\title{
COMPOSIÇÃO FLORÍSTICA DA ARBORIZAÇÃO URBANA DE ANALÂNDIA/SP, BRASIL
}

\author{
FLORISTIC COMPOSITION OF URBAN FORESTRY IN ANALÂNDIA/SP, BRAZIL
}

\author{
Gustavo Torres Galvão da Silva ${ }^{1}$, Nicolli Bruna Cabello², Paulo Henrique Gaem Barbosa ${ }^{3}$, \\ Larissa de Lima Belinazi ${ }^{4}$, José Mauro Santana da Silva ${ }^{5}$, Fiorela Fernanda Mazine Capelo ${ }^{6}$
}

\section{RESUMO}

O bem-estar e o conforto da população em uma cidade são positivamente afetados com a presença de arborização nas vias públicas. Assim, visto que, os estudos sobre arborização urbana são recentes no Brasil e, o município de Analândia apresenta potencial de conservação ambiental, o objetivo desse trabalho foi quantificar a arborização urbana de Analândia (SP) contribuindo para um plano de arborização e melhoria da cidade. Logo, foi realizado um levantamento censitário da arborização urbana em todas as ruas e avenidas do município de Analândia, com o objetivo de conhecer a composição florística, identificar a origem das espécies e detectar possíveis espécies ameaçadas de extinção. Este estudo foi realizado no período de setembro de 2017 a novembro de 2017. Foram amostradas 1.541 árvores distribuídas em 57 espécies pertencentes a 22 famílias. Do total de espécies, quatro estão referidas na Lista Vermelha da IUCN como tendo algum grau de ameaça. A maioria dos indivíduos $(52,76 \%)$ e das espécies (42,86\%) são nativas do Brasil. O índice de Shannon Wiener ( $\left.H^{\prime}\right)$ foi de 3,19, valor alto se comparado a outros municípios do Brasil. Uma grande variedade de espécies foi observada, havendo dominância de poucas espécies na arborização urbana de Analândia.

Palavras-chave: Silvicultura urbana; Diversidade; Gestão urbana.

\section{ABSTRACT}

The well-being and comfort of the population in a city are positively affected by the presence of trees on public roads. Thus, since studies on urban afforestation are recent in Brazil and the municipality of Analândia has potential for environmental conservation, the objective of this study was to quantify the urban afforestation of Analândia (SP) contributing to a plan for afforestation and improvement of the city. So, a census of the urban afforestation in all streets and avenues of the city of Analândia was carried out with the objective of knowing the floristic composition, identifying the origin of the species and detecting possible threatened species. This study was conducted from September through November, 2017. It was sampled 1541 trees distributed in 57 species belonging to 22 families. Out of that 57 species, four (04) integrate the IUCN Red List as having some threatening degree. Most individuals (52,76\%) and species $(42,86 \%)$ are native to Brazil. The Shannon Wiener index $\left(H^{\prime}\right)$ was 3,19 - a high value if compared to other cities of Brazil. A great diversity of species was observed, having dominancy of few species in urban afforestation of Analândia.

Keywords: Urban forestry; Diversity; Urban management.

Recebido em 20.07.2019 e aceito em 02.03.2020

1 Engenheiro Florestal - Universidade Federal de São Carlos, campus Sorocaba/SP. Email: gtorres_galvao@hotmail.com

2 Docente em Engenharia Florestal - Universidade Federal de São Carlos, campus Sorocaba/SP. Email: nicollibruna@gmail.com 3 Engenheiro Florestal. - Universidade Federal de São Carlos, campus Sorocaba/SP. Email: phgaem@gmail.com

4 Docente em Engenharia Florestal - Universidade Federal de São Carlos, campus Sorocaba/SP. Email: larissabeli@hotmail.com

5 Doutor em Irrigação e Drenagem pela Universidade Estadual de São Paulo, campus Botucatu/SP. Professor em Universidade

Federal de São Carlos, campus Sorocaba/SP. Email: josemauro@ufscar.com

6 Doutora em Ciências pela Universidade de São Paulo. Professora em Universidade Federal de São Carlos, campus Sorocaba/SP. Email: fiorela@ufscar.com 


\section{INTRODUÇÃO}

O bem-estar e o conforto da população podem ser afetados por diversos fatores presentes na cidade, dentre eles, a presença de arborização nas vias públicas, ou seja, a existência de espécies vegetais em seus espaços físicos, como praças, ruas e jardins. A configuração de uma infraestrutura verde nas cidades pode contribuir com a redução de tensões e impactos negativos à população, principalmente devido à função de regulação microclimática em que as mesmas fornecem aos locais. A arborização de vias públicas ou urbanas consiste em trazer para as cidades - pelo menos simbolicamente - um pouco do ambiente natural e do verde das matas, com a finalidade de satisfazer as necessidades mínimas do ser humano. Assim, a busca pela maior qualidade de vida para os cidadãos, tem se tornado um dos focos principais em discussões ambientais urbanas (WESTPHAL, 2000; KOLBE et al., 2016; COSTA et al., 2017; SARTORI et al., 2019; WANG et al., 2019).

A composição arbórea de uma cidade apresenta inúmeras funções, as quais desempenham papel importante devido aos serviços ecossistêmicos que ela desempenha, como por exemplo o aumento da riqueza em biodiversidade, o aumento de sombreamento, amenização da poluição atmosférica e sonora, melhorando a qualidade do ar. Do ponto de vista estético, contribui através das qualidades visuais (cor, forma, textura), emoldurando ruas e avenidas, contribuindo para reduzir o efeito agressivo das construções que dominam a paisagem urbana. E, por último, embora difícil de quantificar, diz respeito ao aspecto psicológico, com a satisfação que o homem sente através do contato com a vegetação e com o ambiente que ela cria (LOCKE; BAINE, 2015; BURIVALOVA et al., 2015; KOLBE et al., 2016; COSTA et al., 2017; PIRES et al., 2019; SARTORI et al., 2019).

Os estudos sobre arborização urbana são recentes no Brasil. Nesse contexto, Analândia é uma cidade que apresenta um potencial de conservação ambiental em virtude da formação florestal presente. Analândia tem a maior parte do território composto por pastagem e cultura de cana-de-açúcar (Figura 1), no entanto, considerando a cobertura vegetal, há o predomínio de áreas de reflorestamento (4,99\% da área do município), seguida por regiões de vegetação secundária, ou também chamada de capoeira (4,84\%), florestas densas $(3,89 \%)$, cerrado $(3,42 \%)$, cerradão $(0,82 \%)$ e uma pequena porção de vegetação de várzea, localizadas ao longo dos cursos d'água correspondendo a $0,37 \%$ da área municipal (OLIVEIRA; TAVARES, 2019). 


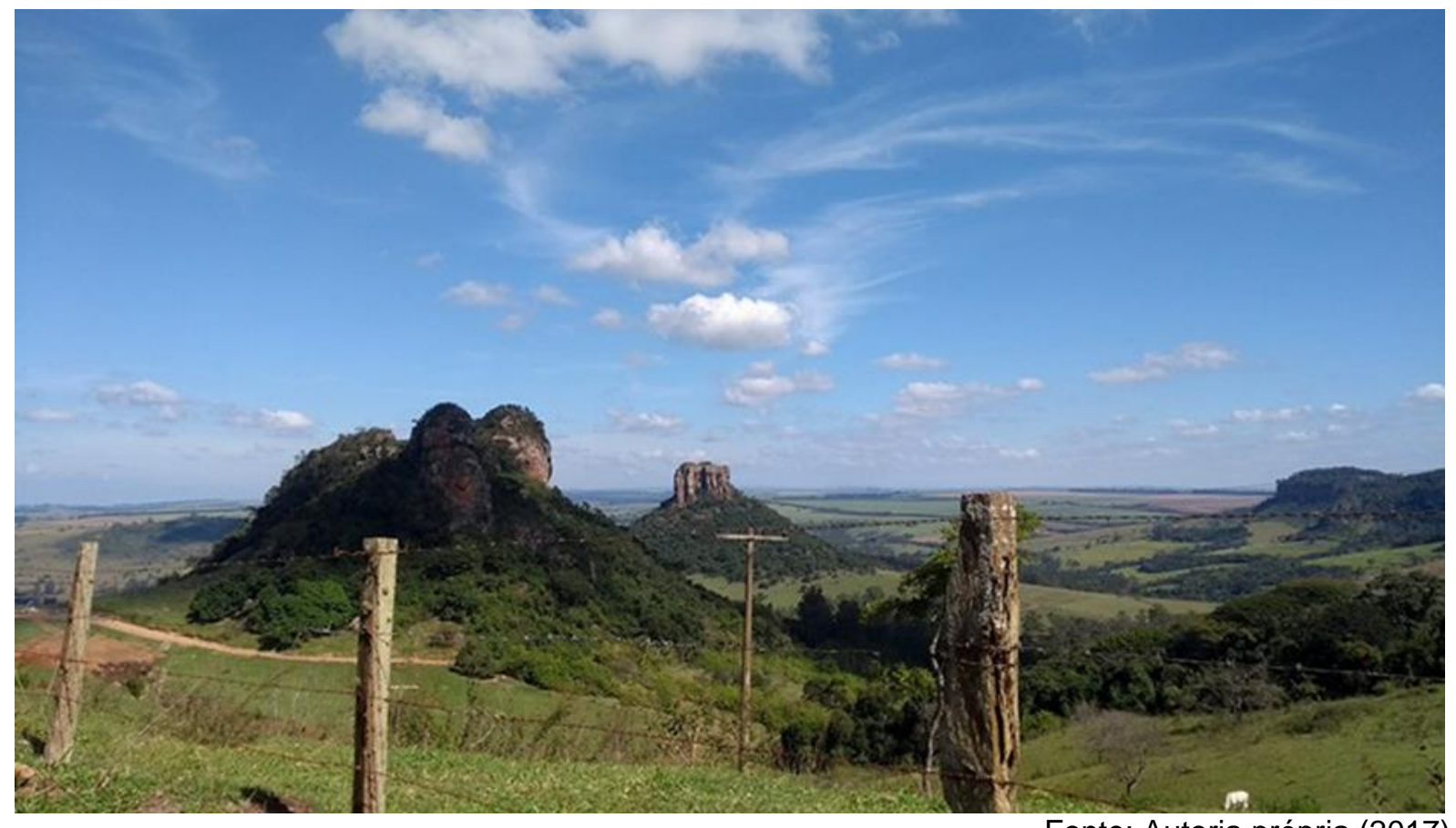

Figura 1. Belezas naturais do município de Analândia, Estado de São Paulo, Brasil

Fonte: Autoria própria (2017) Figure 1. Natural beauties of the municipality of Analândia, State of São Paulo, Brazil

Por estar situada entre a Depressão Periférica Paulista e as Cuestas Basálticas, Analândia é constituída por uma paisagem de grande beleza visual, rica em quedas d'água, morros, nascentes e cavernas, destacando-se como uma cidade de grande potencial turístico (ALVARENGA; SOUZA, 1998; LEITE, 2002). Em busca de melhores condições de vida urbana, o planejamento e administração das cidades envolvem análises das estruturas e suas funções, através de óticas econômicas, sociais e ambientais. Assim, delineia-se o objetivo desse estudo no qual se busca quantificar a arborização urbana de Analândia (SP) contribuindo para um plano de arborização e melhoria da cidade.

\section{MATERIAL E MÉTODOS}

\section{Área de estudo}

A cidade de Analândia situa-se aproximadamente a $684 \mathrm{~m}$ de altitude, em latitude de $22^{\circ} 08^{\prime} \mathrm{S}$ e longitude de $47^{\circ} 40^{\prime} \mathrm{W}$, com uma população de 4.672 habitantes (IBGE, 2014), localizada no centro-leste do Estado de São Paulo. Seus limites territoriais são as cidades de Descalvado ao norte, Corumbataí e Itirapina ao sul, Pirassununga e Santa Cruz da Conceição ao leste e São Carlos a oeste. Seus principais acessos são pelas rodovias Washington Luiz (SP-310) e Anhanguera (SP-330), interligadas pela SP-225, onde se encontra a cidade (Figura 2). 


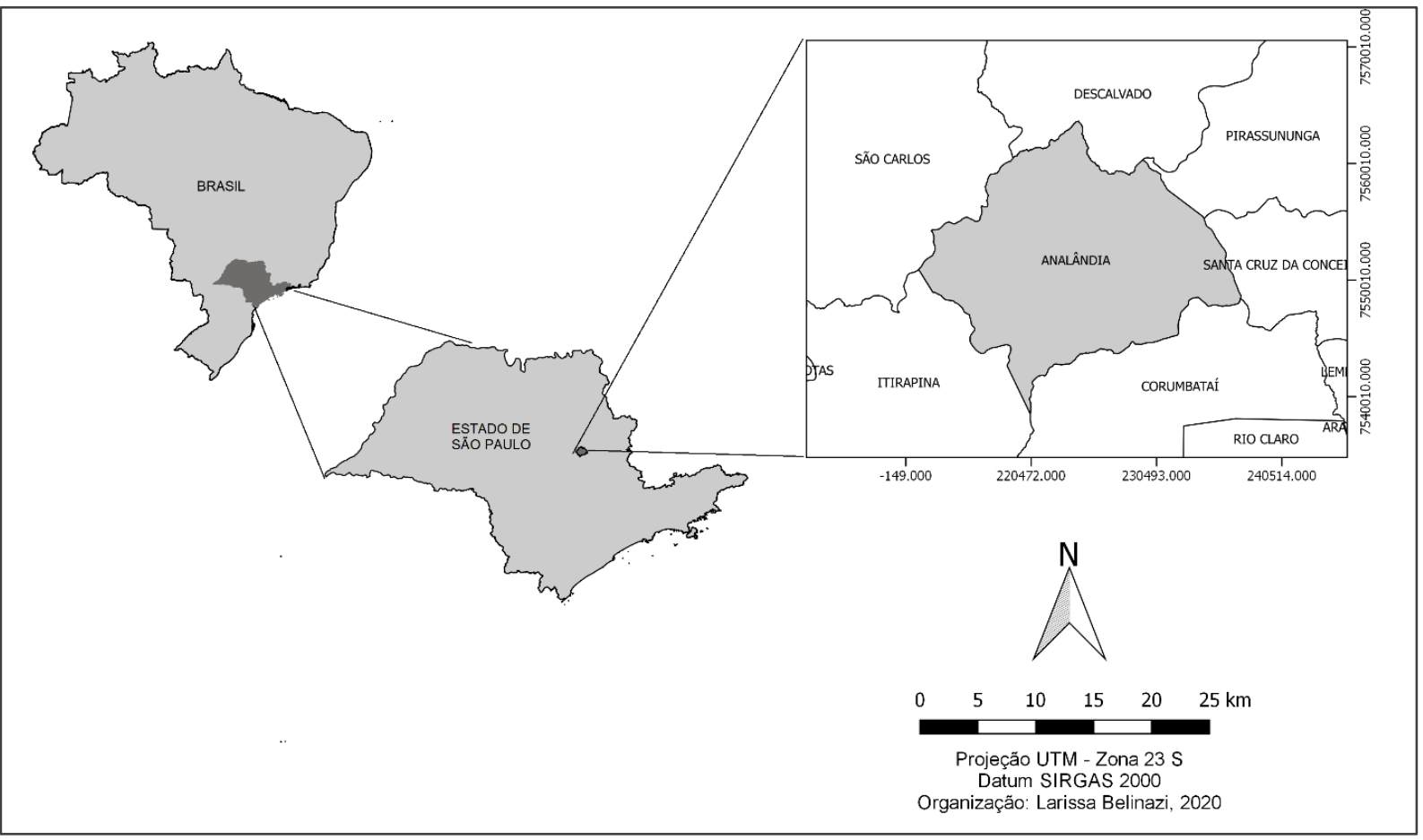

Figura 2. Localização e indicação do município de Analândia, Estado de São Paulo, Brasil Figure 2. Location and indication of the municipality of Analândia, State of São Paulo, Brazil

\section{Coleta e análise de dados}

A coleta de dados foi realizada no período de setembro de 2017 a Outubro de 2017 por uma equipe de sete pessoas, alunos do curso de Engenharia Florestal da UFSCar campus Sorocaba, sob orientação de pesquisadores especialistas, autores deste trabalho.

Foram identificados todos os indivíduos localizados nas ruas e avenidas, plantados em calçadas e canteiros centrais no município de Analândia a partir de observação e coleta de exemplares para consulta, os dados foram anotados em planilhas para posterior checagem. Não foram incluídos neste estudo indivíduos arbóreos localizados em praças e parques urbanos, tampouco os de fragmentos de vegetação nativa.

De todos os indivíduos que levantaram dúvidas, foram coletados material botânico e/ou registros fotográficos do indivíduo inteiro, de detalhes dos ramos, casca, flores e frutos para posterior identificação.

A identificação das espécies foi realizada através da consulta à bibliografia, consulta à especialistas e comparação em herbário (especialmente o herbário SORO, da Universidade Federal de São Carlos, campus Sorocaba). A grafia de todas espécies foi checada na base de dados da Flora do Brasil 2020 (FLORA DO BRASIL, 2020).

A origem (nativa, exótica ou subespontânea) das espécies foram consultados na Lista de Espécies da Flora do Brasil (CARDOSO-LEITE et al., 2019). Além disso, verificou-se a inclusão das espécies na Lista Vermelha de Espécies Ameaçadas da IUCN (2019). 
Logo após a checagem dos nomes, foram realizados os cálculos dos seguintes parâmetros: (a) densidade relativa (DR): $\mathrm{n}^{0}$ indivíduos da espécie/ $\mathrm{n}^{-}$total de indivíduos amostrados; (b) Porcentagem de indivíduos e de espécies nativas (N), exóticas (E), subespontâneas (SUB) e não classificadas (NC); (c) índice de diversidade de espécies de Shannon Wiener $\left(\mathrm{H}^{\prime}\right)$ : - $\Sigma(\mathrm{n}$ i $/ \mathrm{N})$ In $(\mathrm{n}$ i $/ \mathrm{N})$, onde $\mathrm{n}$ i é número de indivíduos de uma dada espécie e $\mathrm{N}$ é número total de indivíduos amostrados.

\section{RESULTADOS E DISCUSSÃO}

Todas as ruas do Município de Analândia foram percorridas, totalizando 45 ruas. Ao todo foram amostrados 1.541 indivíduos, sendo 55 espécies, incluindo árvores e palmeiras. Todos os indivíduos foram identificados ao nível de espécie e calculado a frequência absoluta, como observado na Tabela 1.

Tabela1. Espécies e famílias amostradas no levantamento da arborização urbana de Analândia-SP, Brasil Table 1. Species and families sampled in the urban afforestation survey of Analândia, SP, Brazil

\begin{tabular}{|c|c|c|c|c|c|}
\hline Famílias & Nome científico & Nome Popular & № & $\begin{array}{l}\text { DR } \\
(\%)\end{array}$ & 0 \\
\hline Anacardiaceae & Schinus molle L. & Chorão & 168 & 10,90 & $0 \mathrm{~N}$ \\
\hline Fabaceae & Poincianella pluviosa (DC.) L.P.Queiroz & Sibipiruna & 156 & 10,12 & $2 \mathrm{~N}$ \\
\hline Myrtaceae & Callistemon viminalis G. Don ex Loud & Escovinha-de-garrafa & 135 & 8,76 & $E$ \\
\hline Myrtaceae & Eugenia involucrata DC. & Cereja-do-mato & 125 & 8,11 & $\mathrm{~N}$ \\
\hline Rutaceae & Murraya paniculata (L.) Jack & Falsa-murta & 117 & 7,59 & $E$ \\
\hline Fabaceae & Bauhinia variegata $L$. & Pata de vaca & 91 & 5,91 & E \\
\hline Anacardiaceae & Mangifera indica L. & Mangueira & 81 & 5,26 & $E$ \\
\hline Arecaceae & Syagrus romanzoffiana (Cham.) Glassman & Palmeira & 71 & 4,61 & SUB \\
\hline Anacardiaceae & Schinus terebinthifolia Raddi & Aroeira-pimenteira & 64 & 4,15 & $\mathrm{~N}$ \\
\hline Bignoniaceae & $\begin{array}{l}\text { Handroanthuss chrysotrichus (Mart. Ex DC.) } \\
\text { Mattos }\end{array}$ & Ipê amarelo & 58 & 3,76 & $N$ \\
\hline Apocynaceae & Nerium oleander $\mathrm{L}$. & Espirradeira & 43 & 2,79 & $E$ \\
\hline Melastomataceae & Pleroma granulosa (Desr.) D. Don & Quaresmeira & 32 & 2,08 & $N$ \\
\hline Chrysobalanaceae & Licania tomentosa (Benth.) Fritsch & Oiti & 31 & 2,01 & $\mathrm{~N}$ \\
\hline Bignoniaceae & Tabebuia roseoalba (Ridl.) Sandwith & Ipê branco & 28 & 1,82 & $\mathrm{~N}$ \\
\hline Bignoniaceae & Spathodea campanulata P. Beauv. & Espatódia & 23 & 1,49 & $E$ \\
\hline Malvaceae & Hibiscus rosa-sinenseL. & Hibisco & 23 & 1,49 & $E$ \\
\hline Combretaceae & Terminalia catappa L. & Chapéu do sol & 22 & 1,43 & SUB \\
\hline Muntigiaceae & Muntingia calabura $\mathrm{L}$. & Calabura,tamuã & 22 & 1,43 & $N$ \\
\hline Moraceae & Ficus benjamina L. & Figueira & 20 & 1,3 & $\mathrm{NC}$ \\
\hline Bignoniaceae & Tecoma stans (L.) Juss. Ex Kunth & Ipê falso & 18 & 1,17 & $\mathrm{~N}$ \\
\hline Fabaceae & Tipuana tipu (Benth.) Kuntze & Tipuana & 18 & 1,17 & SUB \\
\hline Verbenaceae & Duranta repens L. & Pingo de ouro & 18 & 1,17 & $E$ \\
\hline Arecaceae & Cocos nucifera L. & Coqueiro & 16 & 1,04 & SUB \\
\hline Myrtaceae & Syzygium cumini (L.) Skeels & Jambolão & 15 & 0,97 & SUB \\
\hline Fabaceae & Caesalpinia pulcherrima (L.) Sw & Flamboyant de jardim & 14 & 0,91 & SUB \\
\hline Myrtaceae & Eugenia uniflora L. & Pitanga & 14 & 0,91 & $N$ \\
\hline
\end{tabular}




\begin{tabular}{|c|c|c|c|c|c|}
\hline Myrtaceae & Psidium guajava L. & Goiabeira & 11 & 0,71 & SUB \\
\hline Melastomataceae & Pleroma mutabilis (Vell.) Triana & Manacá da serra & 9 & 0,58 & $\mathrm{~N}$ \\
\hline Bignoniaceae & $\begin{array}{l}\text { Handroanthus impetiginosus (Mart. Ex DC.) } \\
\text { Mattos }\end{array}$ & Ipê roxo & 7 & 0,45 & $\mathrm{~N}$ \\
\hline Meliaceae & Azadirachta indica & $\mathrm{Nim}$ & 7 & 0,45 & $E$ \\
\hline Meliaceae & Cedrela fissilis Vell. & Cedro & 7 & 0,45 & $\mathrm{~N}$ \\
\hline Rosaceae & Eriobotrya japonica & Nespeira & 7 & 0,45 & SUB \\
\hline Anacardiaceae & Schinus molle L. & Figo chorão & 7 & 0,004 & $E$ \\
\hline Araliaceae & Schefflera ariegate (Hayata) Merr. & Scheflera & 6 & 0,39 & $\mathrm{E}$ \\
\hline Araucariaceae & Araucaria angustifolia & Pinheiro do paraná & 6 & 0,39 & $\mathrm{~N}$ \\
\hline Rutaceae & Citrus sp. & Limão & 6 & 0,39 & E \\
\hline Cupressaceae & Cupressus sempervirens L. & Cepreste & 5 & 0,32 & $\mathrm{E}$ \\
\hline Fabaceae & Caesalpinia pulcherrima (L.) Sw & Flamboyanzinho & 5 & 0,32 & $E$ \\
\hline Moraceae & Morus nigra L. & Amora & 5 & 0,32 & $E$ \\
\hline Pinaceae & Pinus sp. & Pinheiro & 5 & 0,32 & $\mathrm{E}$ \\
\hline Bixaceae & Bixa orellana $\mathrm{L}$. & Urucum & 3 & 0,19 & $\mathrm{~N}$ \\
\hline Fabaceae & Hymenaea courbaril L. & Jatobá & 3 & 0,19 & $\mathrm{~N}$ \\
\hline Fabaceae & $\begin{array}{l}\text { Paubrasilia echinata (Lam.) E. Gagnon, H.C. } \\
\text { Lima \& G.P. Lewis }\end{array}$ & Pau brasil & 3 & 0,19 & $\mathrm{~N}$ \\
\hline Malvaceae & Ceiba ariegat (A.St.-Hil.) Ravenna & Paineira & 3 & 0,19 & $\mathrm{~N}$ \\
\hline Fabaceae & Inga sp. & Ingá & 2 & 0,13 & $\mathrm{~N}$ \\
\hline Asteraceae & $\begin{array}{l}\text { Moquiniastrum polymorphum (Less.) G. } \\
\text { Sancho }\end{array}$ & Cambará & 1 & 0,06 & $\mathrm{~N}$ \\
\hline Bignoniaceae & Jacaranda mimosifolia D.Don & Jacarandá & 1 & 0,06 & $\mathrm{E}$ \\
\hline Euphorbiaceae & Croton urucurana Baill. & Sangra d'agua & 1 & 0,06 & $\mathrm{~N}$ \\
\hline Fabaceae & Calliandra brevipes Benth. & Calliandra & 1 & 0,06 & NC \\
\hline Fabaceae & Erythrina speciosa Andrews & Mulungu-do-litoral & 1 & 0,06 & $\mathrm{E}$ \\
\hline Fabaceae & Leucaena leucocephala (Lam.) de Wit & Leucena & 1 & 0,06 & SUB \\
\hline Fabaceae & Mimosa hebecarpa & Angico & 1 & 0,06 & NC \\
\hline Fabaceae & Schizolobium parahyba (Vell.) Blake & Guapuruvu & 1 & 0,06 & $\mathrm{~N}$ \\
\hline Malpighiaceae & Malpighia emarginata DC. & Acerola & 1 & 0,06 & E \\
\hline Myrtaceae & $\begin{array}{l}\text { Myrciaria glazioviana (Kiaersk.) G.M.Barroso } \\
\text { ex Sobral }\end{array}$ & Peludinha & 1 & 0,06 & $\mathrm{~N}$ \\
\hline Sapindaceae & Paullinia arieg & Araçá & 1 & 0,06 & $\mathrm{~N}$ \\
\hline
\end{tabular}

NOTA: $\mathrm{N}^{\circ}=$ número de indivíduos amostrados, $\mathrm{DR}=$ densidade relativa $(\%$ do total de indivíduos amostrados), $\mathrm{O}$ = origem, $\mathrm{N}$ = nativa, $\mathrm{E}=$ exótica, $\mathrm{SUB}$ = subespontânea, $\mathrm{NC}$ = não classificada.

No presente levantamento foi observada dominância de duas espécies (Tabela 1), Schinus molle L (Chorão) e Poincianella pluviosa (DC.) L.P.Queiroz (Sibipiruna). Segundo Santamour Júnior (2002), recomenda-se que não se utilize mais do que $10 \%$ a $15 \%$ de árvores de uma mesma espécie em arborização urbana para evitar a propagação de pragas e doenças entre as plantas, além de se obter um planejamento adequado da arborização. Assim, de acordo com a frequência observada das respectivas espécies, 10,90\% e 10,12\%, a arborização urbana se encontra dentro do estabelecido pelo autor.

$\mathrm{Na}$ Figura 3 pode-se observar a presença de flores de ipê amarelo (Handroanthuss chrysotrichus), falsa murta (Murraya paniculata), Calliandra (Calliandra brevipes) e pata de vaca (Bauhinia variegata), tendo forte contribuição nos serviços ecossistêmicos do local. 


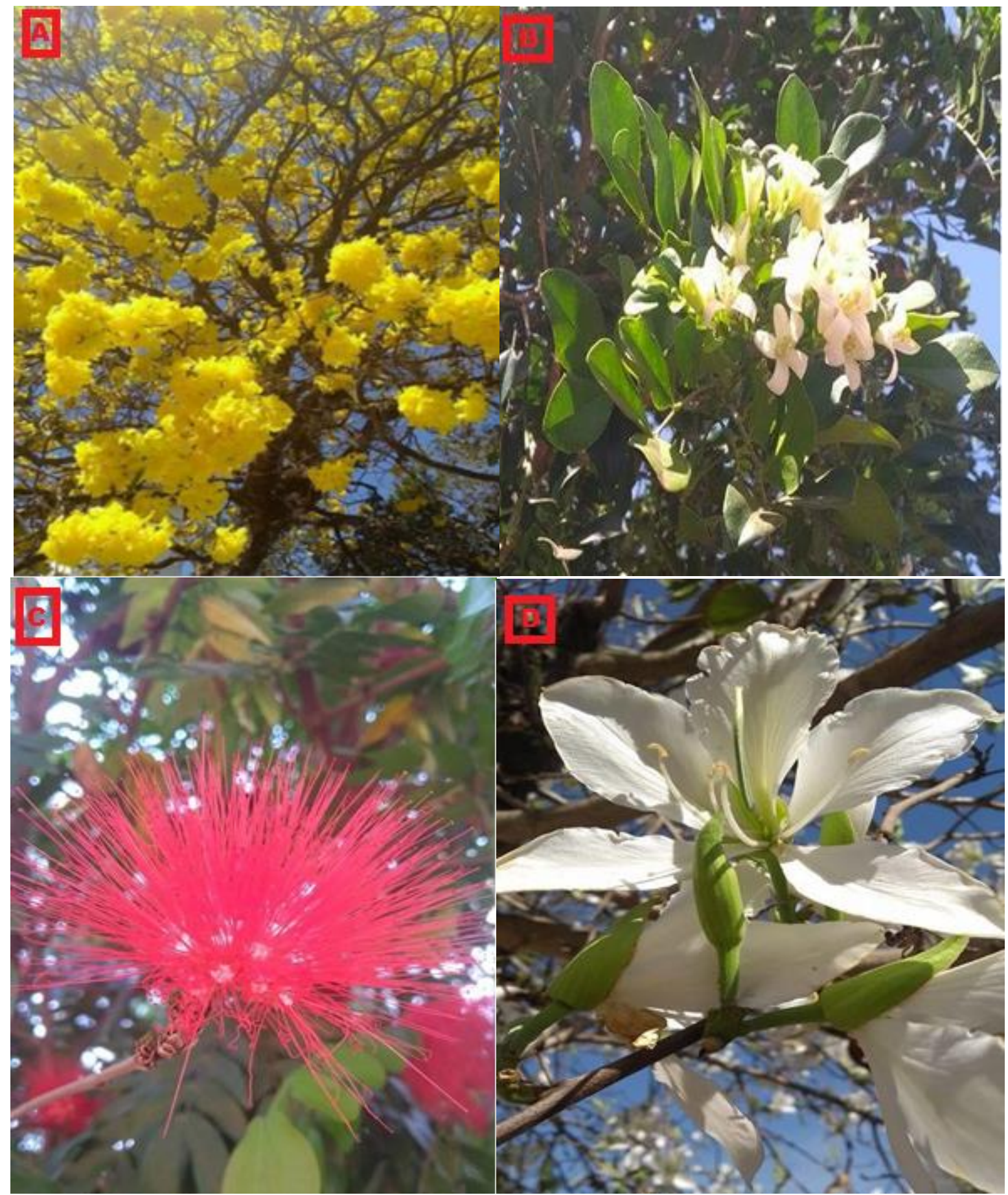

Figura 3. Espécies frequentemente encontradas na arborização urbana de Analândia, SP, Brasil. Sendo a letra A o Ipê Amarelo (Handroanthuss chrysotrichus), a letra B a Falsa Murta (Murraya paniculata), a letra C a Calliandra (Calliandra brevipes) e a letra D a Pata de Vaca (Bauhinia variegata)

Figure 3. Species frequently found in the urban afforestation of Analândia, SP, Brazil. Letter A is Golden trumpet tree (Handroanthuss chrysotrichus), letter B is Murraya ariegate Orange jessamine (Murraya paniculata), letter $\mathrm{C}$ is Pink powderpuff (Calliandra brevipes) and letter D is Orchid tree (Bauhinia variegata) 
As famílias com maior número de espécies foram Fabaceae (14), Myrtaceae (8) e Bignoniaceae (6) que juntas somaram $47,70 \%$ do total de espécies amostradas, mas a família com maior representatividade foi Anacardiaceae com 20,31\% devido à grande presença de Schinus molle (chorão), seguida de Myrtaceae com 19,66\% e em terceiro Fabaceae com 19,27\%, sendo Bauhinia variegata (pata de vaca) a espécie com maior número de indivíduo. Já dentre as Myrtaceae, predominou Callistemon viminalis (escovinha de garrafa), sendo que no bairro CDHU a presença dela foi de $77,77 \%$ das árvores amostradas em todo o bairro. $\mathrm{A}$ família Bignoniaceae é representada em sua grande maioria pelos ipês, que em geral são espécies nativas (Figuras 4 e 5 ).

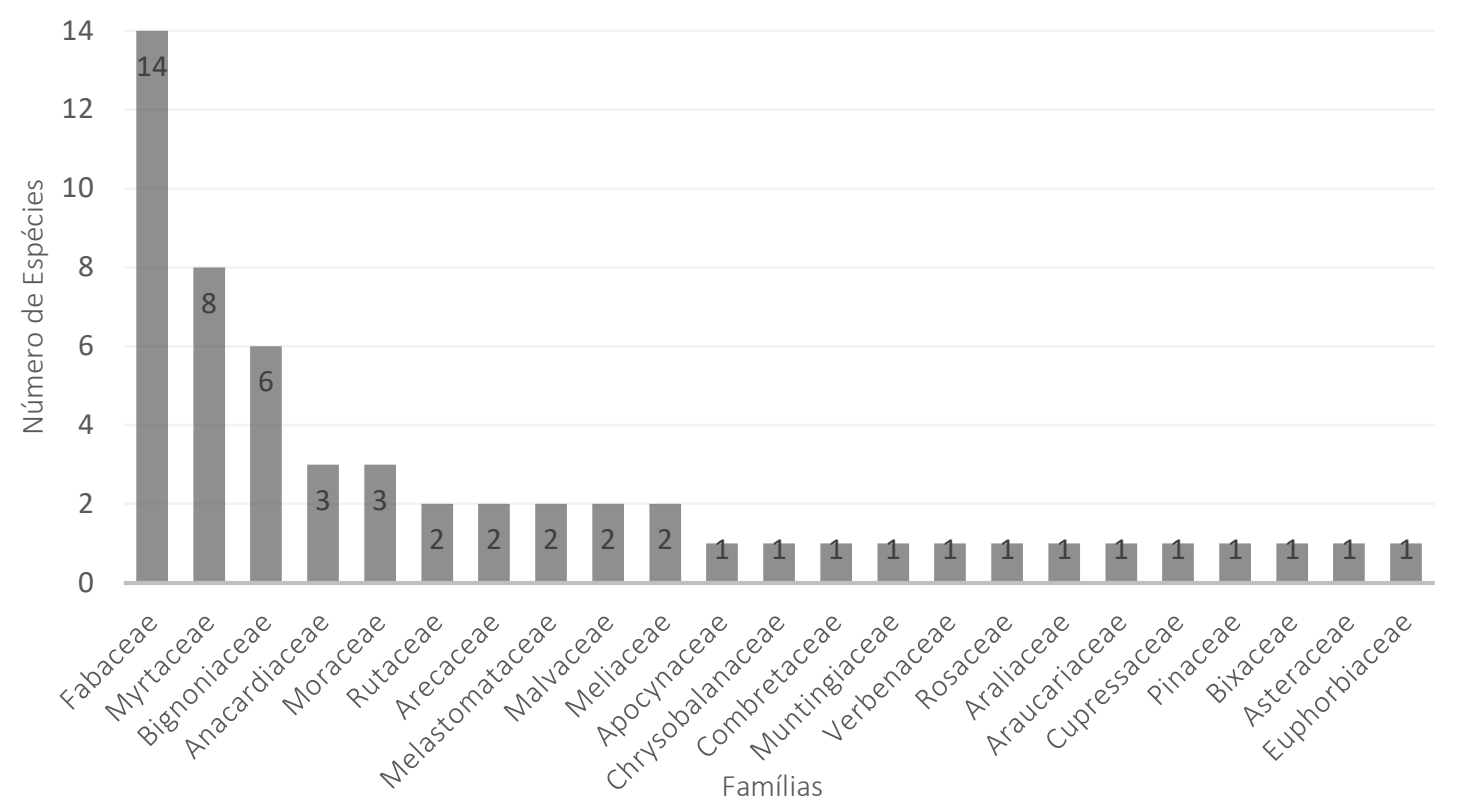

Figura 4. Número de indivíduos por famílias amostradas no censo da arborização urbana de Analândia, $\mathrm{SP}$, Brasil

Figure 4. Number of individuals by families sampled in the urban afforestation census of Analândia, SP, Brazil

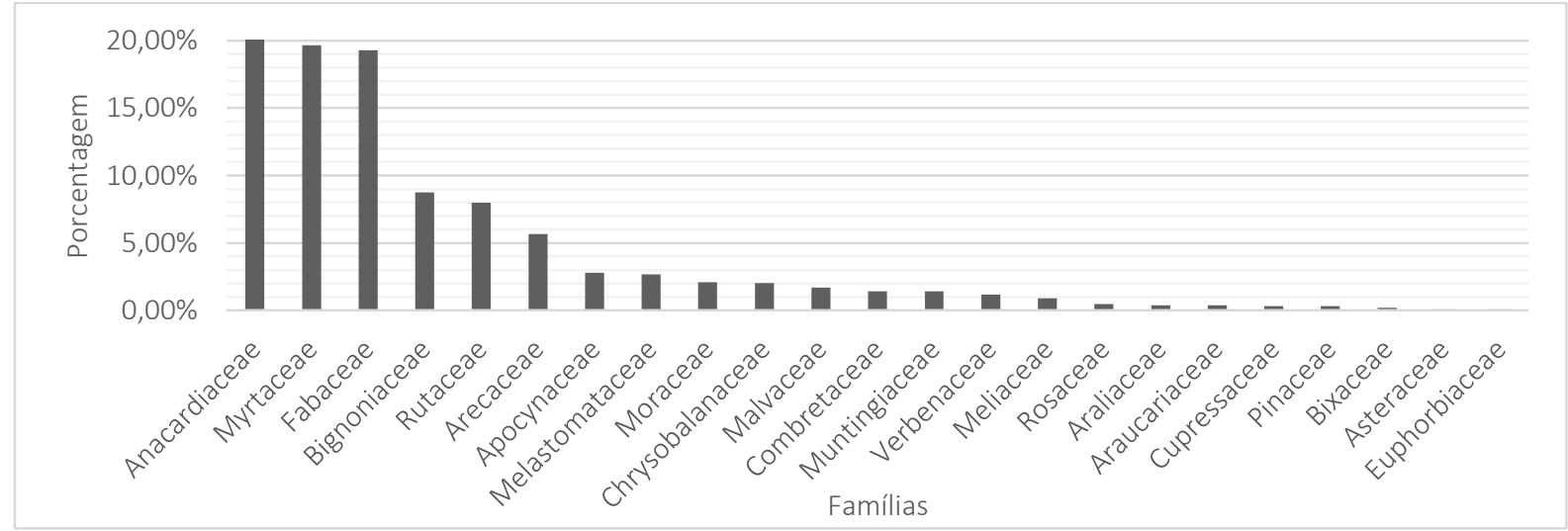

Figura 5. Porcentagem de espécies por famílias amostradas no censo da arborização urbana de Analândia, SP, Brasil

Figure 5. Percentage of species by families sampled in the urban afforestation census of Analândia, SP, Brazil 
Cardoso-Leite et al. (2019) também encontraram os maiores números de espécies de Fabaceae, Myrtaceae e Bignoniaceae em Sorocaba/SP, indicando o uso intensivo de espécies destas famílias na arborização urbana no interior de São Paulo. A dominância de poucas espécies também foi observada por Rocha et al. (2014) em Nova Iguaçu/RJ, e em Assis/SP (ROSSATTO; TSUBOY; FREI, 2008), indicando que este é um padrão recorrente em algumas regiões do Brasil. Ao contrário de Águas de São Pedro/SP (BORTOLETO et al., 2019) que também se trata de uma estância, no qual teve predomínio de Fabaceae, Moraceae e Arecaceae respectivamente.

Neste presente trabalho, 42,86\% (24) das espécies amostradas são nativas do Brasil, 35,71\% (20) são exóticas e 16,07\% (9) subespontâneas (Figura 6).

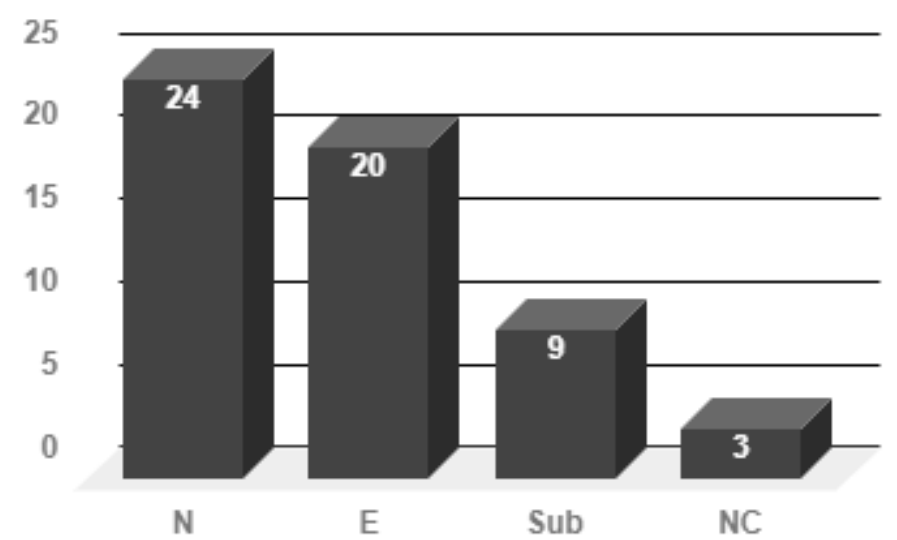

Figura 6. Porcentagem de espécies nativas (N), exóticas (E), subespontâneas (SUB) e não classificadas (NC), amostradas no levantamento da arborização urbana de Analândia, SP, Brasil

Figure 6. Percentage of native (N), exotic $€$, subspontaneous (SUB) and unclassified (NC) species, sampled in the urban afforestation survey of Analândia, SP, Brazil

Em relação ao número de indivíduos, 52,76\% (813) são de espécies nativas, 39,13\% (603) exóticas e 6,68\% (103) subespontâneas (Figura 7).

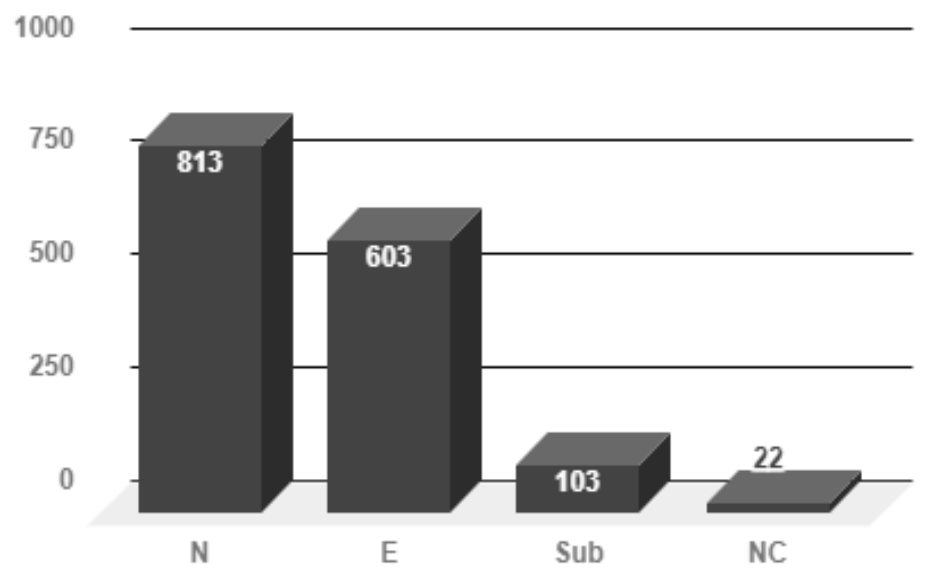

Figura 7. Porcentagem de indivíduos nativos (N), exóticos (E), subespontâneos (SUB) e não classificados (NC), amostrados no levantamento da arborização urbana de Analândia, SP, Brasil

Figure 7. Percentage of native (N), exotic $€$, subspontaneous (SUB) and unclassified (NC) individuals, sampled in the urban afforestation survey of Analândia, SP, Brazil 
Assim, tanto para espécies quanto para número de indivíduos houve predomínio de espécies nativas. Cardoso-Leite et al. (2019) encontraram uma proporção semelhante de espécies e indivíduos nativos para o município de Sorocaba/SP, assim como Rocha et al. (2014) na arborização urbana de Assis/SP. O que é incomum em cidades brasileiras onde geralmente predominam espécies exóticas, como nos casos de Assis/SP com 61,82\%, de Cosmópolis/SP com o 57,5\% e Águas de São Pedro/SP com 61,33\% (BORTOLETO et al., 2019; DE PAIVA, 2019; ROSSATTO; TSUBOY; FREI, 2019).

Segundo a Lista Vermelha da União Internacional para a Conservação da Natureza e dos Recursos Naturais (IUCN Red List, 2019), quatro espécies amostradas apresentam algum grau de ameaça de extinção, sendo Jacaranda mimosifolia D.Don e Cedrela fissilis Vell. na categoria vulnerável (VU), e Paubrasilia echinata (Lam.) E.Gagnon, H.C.Lima \& G.P.Lewis em perigo (EN) e Araucaria angustifolia (Cham.) Glassman em perigo crítico (CR). O número de espécies ameaçadas encontrado foi três vezes menor em relação ao referido por CardosoLeite et al. (2019) para Sorocaba/SP.

Em Analândia registrou-se uma árvore para cada 3 cidadãos, ou 0,329 árvores por indivíduo, considerando os dados de 2014 do IBGE onde a população registrada foi de 4.672 habitantes. Cabe ressaltar que o presente estudo não analisou as praças, parques urbanos e remanescentes de floresta nativa no município, o que certamente aumentaria o número de árvores por habitante na cidade (IBGE, 2014).

O índice de Shannon Wiener $\left(\mathrm{H}^{\prime}\right)$ calculado foi de 3,19, valor próximo ao encontrado em Sorocaba/SP, de 3,73 (CARDOSO-LEITE et al., 2019). No entanto, maior quando comparado com algumas regiões do estado de São Paulo, como na Flona Nacional de Ipanema, que apresentou um índice de 2,27 (BATAGHIN; BARROS; PIRES, 2010) ou no município de São José do Rio Preto, com um índice de 2,77 (FERNANDES et al., 2018).

\section{CONCLUSÕES}

De acordo com o número de árvores amostradas na arborização urbana da cidade de Analândia, precisamente 1.541 indivíduos, conclui-se que há uma diversidade de espécies de acordo com o índice de Shannon Wieneer $\left(H^{\prime}\right)$, pois este se apresenta maior quando comparado à outras regiões de São Paulo.

Pode-se concluir também que o município apresenta uma arborização urbana bem planejada, considerando o número de árvores amostradas, a diversidade de espécies utilizadas (55 espécies) e a porcentagem de espécies nativas (42,86\%). No entanto, há baixa presença de espécies ameaçadas, sendo necessários outros estudos que avaliem a vegetação e 
distribuição nas diferentes regiões da cidade para realizar um planejamento futuro que investisse na arborização da cidade.

\section{REFERÊNCIAS}

ALVARENGA, S. R.; SOUZA, M. P. Análise das áreas de proteção ambiental (APAs) como instrumento para manutenção da qualidade dos recursos hídricos: o caso da APA Corumbataí - SP, 1998, Gramado. Anais... Gramado: Simpósio Internacional sobre Gestão de Recursos Hídricos, 1998.

BATAGHIN, F. A.; BARROS, F.; PIRES, J. S. R. Distribuição da comunidade de epífitas vasculares em sítios sob diferentes graus de perturbação na Floresta Nacional de Ipanema, São Paulo, Brasil. Brazilian Journal of Botany, São Paulo, v. 33, n. 3, p. 501-512, 2010.

BORTOletO, S.; da SILVA FILHO, D. F.; SOUZA, V. C.; de PINHO FERREIRA, M. A.; POLIZEL, J. L.; RIBEIRO, R. D. C. S. Composição e distribuição da arborização viária da estância de Águas de São Pedro-SP. Revista da Sociedade Brasileira de Arborização Urbana, Piracicaba, v. 2, n. 3, p. 32-46, 2019.

CARDOSO-LEITE, E.; de FARIA, L. C.; CAPELO, F. F. M.; TONELLO, K. C.; CASTELLO, A. C. D. Composição florística da arborização urbana de Sorocaba/SP, Brasil. Revista da Sociedade Brasileira de Arborização Urbana, Piracicaba, v.9, n.1, p.133-150, 2019.

COSTA, C. F.; FONSECA, R. S.; de ALMEIDA, D. B.; de OLIVEIRA, M. S.; dos SANTOS OLIVEIRA, D.; BRAGA, J. H. P. Espécies utilizadas na arborização em praças do município de Caxias, Maranhão. Revista da Sociedade Brasileira de Arborização Urbana, Piracicaba, v. 12, n. 1, p. 65-78, 2017.

BURIVALOVA, Z.; LEE, T. M.; GIAM, X.; ŞEKERCIOĞLU, Ç. H.; WILCOVE, D. S.; KOH, L. P. Avian responses to selective logging shaped by species traits and logging practices. Proceedings of the Royal Society B: Biological Sciences, Londres, v. 282, n. 1808, 2015.

DE PAIVA, A. V. Aspectos da arborização urbana do Centro de Cosmópolis-SP. Revista da Sociedade Brasileira de Arborização Urbana, Piracicaba, v. 4, n. 4, p. 17-31, 2019.

FERNANDES, C. J.; FERRAZ, M. V.; GIMENES, R.; PEREIRA, S. T. S.; PIVETTA, K. F. L. Trees description in the main square of São José do Rio Preto, São Paulo State, Brazil. Ornamental Horticulture, Viçosa, v. 24, n. 4, p. 334-340, 2018.

FLORA DO BRASIL 2020. Jardim Botânico do Rio de Janeiro. Base de dados. Disponível em: <http://floradobrasil.jbrj.gov.br> Acesso em: 23 jul. 2019.

IBGE - INSTITUTO BRASILEIRO DE GEOGRAFIA E ESTATÍSTICA. Estimativas da população residente no Brasil e Unidades da Federação com data de referência em 1 de julho de 2014. Diretoria de Pesquisas, Coordenação de População e Indicadores Sociais - Rio de Janeiro, 2014. Disponível em: <ftp://ftp.ibge.gov.br/Estimativas_de_Populacao/Estimativas _2014/estimativas_2014_TCU.pdf\&gt> Acesso em: 18 jul. 2019.

IUCN 2019. The IUCN Red List of Threatened Species. Versão 2019-3. Lista vermelha. Disponível no site: <http://www.iucnredlist.org> Acesso em: 18 jul. 2019. 
KOLBE, S. E.; MILLER, A. I.; CAMERON, G. N.; CULLEY, T. M. Effects of natural and anthropogenic environmental influences on tree community composition and structure in forests along an urban-wildland gradient in southwestern Ohio. Urban ecosystems, Nova York, v. 19, n. 2, p. 915-938, 2016.

LEITE, S. S. Análise ambiental da área do morro do Cuscuzeiro (Analândia, São Paulo), como subsídio ao planejamento do ambiente local. São Carlos, 2002. 161f. Dissertação (Mestrado em Ecologia e Recursos Naturais) - Universidade Federal de São Carlos, São Carlos, 2002.

LOCKE, D. H.; BAINE, G. The good, the bad, and the interested: how historical demographics explain present-day tree canopy, vacant lot and tree request spatial variability in New Haven, CT. Urban Ecosystems, Nova York, v. 18, n. 2, p. 391-409, 2015.

OLIVEIRA, G. S.; TAVARES, A. A. Levantamento e caracterização das variáveis utilizadas em estudos sobre arborização urbana. Revista da Sociedade Brasileira de Arborização Urbana, Piracicaba, v. 7, n. 3, p. 75-87, 2019.

PIRES, N. A. M. T.; da SILVA MELO, M.; DE OLIVEIRA, D. E.; XAVIER-SANTOS, S. A arborização urbana do município de Goiandira/GO - Caracterização quali-quantitativa e propostas de manejo. Revista da Sociedade Brasileira de Arborização Urbana, Piracicaba, v. 5, n. 3, p. 185-205, 2019.

ROCHA, R. T. D.; LELES, P. S. D. S.; OLIVEIRA NETO, S. N. D. Arborização de vias públicas em Nova Iguaçu, RJ: o caso dos bairros Rancho Novo e Centro. Revista Árvore, Viçosa, v.28, n.4, p. 599-607, 2014.

ROSSATTO, D. R.; TSUBOY, M. S. F.; FREI, F. Arborização urbana na cidade de Assis-SP: uma abordagem quantitativa. Revista da Sociedade Brasileira de Arborização Urbana, Piracicaba, v.3, n.3, p.1-16, 2008.

ROSSATTO, D.; TSUBOY, M. S. F.; FREI, F. Arborização urbana na cidade de Assis-SP: uma abordagem quantitativa. Revista da Sociedade Brasileira de Arborização Urbana, Piracicaba, v. 3, n. 3, p. 1-16, 2019.

SANTAMOUR JÚNIOR, F. S. Trees for urban planting: diversity unifomuty, and common sense. Agriculture Research Service, Washington: U.S. National Arboretum, 2002.

SARTORI, R. A.; MARTINS, G. A. C.; ZAÚ, A. S.; BRASIL, L. S. C. Urban afforestation and favela: A study in A community of Rio de Janeiro, Brazil. Urban Forestry \& Urban Greening, Alemanha, v. 40, p. 84-92, 2019.

WANG, W.; ZHANG, B.; ZHOU, W.; LV, H.; XIAO, L.; WANG, H.; DU, H.; HE, X. The effect of urbanization gradients and forest types on microclimatic regulation by trees, in association with climate, tree sizes and species compositions in Harbin city, northeastern China. Urban Ecosystems, Nova York, v. 22, n. 2, p. 367-384, 2019.

WESTPHAL, M. F. O Movimento Cidades/Municípios Saudáveis: um compromisso com a qualidade de vida. Ciência e saúde coletiva, Rio de Janeiro, v.5, n.1, p.39-51, 2000. 